\title{
Differential Regional Distribution of Juxtacortical White Matter Signal Abnormalities in Aging and Alzheimer's Disease
}

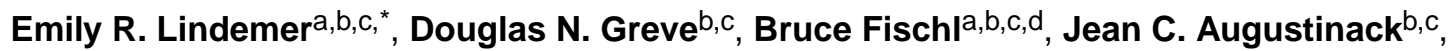 \\ and David H. Salat ${ }^{\mathrm{b}, \mathrm{c}, \mathrm{e}}$ for the Alzheimer's Disease Neuroimaging Initiative ${ }^{\mathbf{1}}$ \\ aHarvard-MIT Division of Health Sciences and Technology, Cambridge, MA, USA \\ ${ }^{b}$ Athinoula A. Martinos Center for Biomedical Imaging, Charlestown, MA, USA \\ 'Department of Radiology, Massachusetts General Hospital, Harvard Medical School, Boston, \\ MA, USA \\ ${ }^{d}$ Computer Science and Artificial Intelligence Laboratory (CSAIL), Massachusetts Institute of \\ Technology (MIT), Cambridge, MA, USA \\ eNeuroimaging Research for Veterans (NeRVe) Center, VA Boston Healthcare System, Boston, \\ MA, USA
}

\section{Abstract}

Background-White matter signal abnormalities (WMSA) (also known as 'hyperintensities') on MRI are commonly seen in normal aging and increases have been noted in Alzheimer's disease (AD), but whether there is a spatial specificity to these increases is unknown.

Objective-To discern whether or not there is a spatial pattern of WMSA in the brains of individuals with $\mathrm{AD}$ that differs from those who exhibit cognitively healthy aging.

Method-Structural MRI data from the Alzheimer's Disease Neuroimaging Initiative public database were used to quantify WMSA in 35 regions of interest (ROIs). Regional measures were compared between cognitively healthy older controls (OC; $n=107)$ and individuals with a clinical diagnosis of $\mathrm{AD}(n=127)$. Regional WMSA volume was also assessed in individuals with mild cognitive impairment (MCI; $n=74$ ) who were 6, 12, and 24 months away from AD conversion.

Results-WMSA volume was significantly greater in AD compared to OC in 24 out of 35 ROIs after controlling for age, and nine were significantly higher after normalizing for total WMSA. Regions with greater WMSA volume in AD included rostral frontal, inferior temporal, and inferior parietal WM. In MCI, frontal and temporal regions demonstrated significantly greater WMSA volume with decreasing time-to-AD-conversion.

\footnotetext{
"Correspondence to: Emily R. Lindemer, BSc, Athinoula A. Martinos Center for Biomedical Imaging, 149 13th St. Suite 2301, Charlestown, MA 02129, USA. lindemer@mit.edu.

${ }^{1}$ Data used in preparation of this article were obtained from the Alzheimer's Disease Neuroimaging Initiative (ADNI) database (http:// adni.loni.usc.edu). As such, the investigators within the ADNI contributed to the design and implementation of ADNI and/or provided data but did not participate in analysis or writing of this report. A complete listing of ADNI investigators can be found at: http:// adni.loni.usc.edu/wp-content/uploads/how_to_apply/ADNI_Acknowledgement_List.pdf.

SUPPLEMENTARY MATERIAL

The supplementary material is available in the electronic version of this article: http://dx.doi.org/10.3233/JAD-161057.
} 
Discussion-Individuals with AD have greater regional volume of WMSA compared to OC regardless of age or total WMSA volume. Accumulation of regional WMSA is linked to time to AD conversion in individuals with MCI. These findings indicate WMSA is an important pathological component of AD development.

\section{Keywords}

Aging; Alzheimer's disease; cerebrovascular; hyperintensities; magnetic resonance imaging; mild cognitive impairment; white matter

\section{INTRODUCTION}

Damage to the cerebral white matter (WM), known by several terminologies including leukoaraiosis, is a pathology commonly found in older adults. Magnetic resonance imaging (MRI) is sensitive to the detection of WM damage, which we refer to here as white matter signal abnormalities (WMSA) [1, 2]. Although histopathological studies have indicated a mix of heterogeneous findings that correlate with WMSA including demyelination and gliosis [3, 4], they are often denoted 'white matter hyperintensities of presumed vascular origin' [5,6], and a loss of surrounding vascular integrity is typically indicated as the upstream pathogenic mechanism responsible for their manifestation in both cognitively normal aging as well as in Alzheimer's disease (AD) [4, 7, 8]. The clinical significance of WMSA has been unclear and in some cases this damage appears to be a benign comorbidity of typical aging with a prevalence as high as $80-95 \%$ in older populations $[9,10]$. However, it is also known that WMSA are associated with some degree of cognitive decline in otherwise healthy older adult [11-13], exhibit alterations in imaging properties around the time of a dementia diagnosis [2], and are increased in volume in AD [14, 15], suggesting a more direct link between this damage and the clinical expression of dementia $[9,16-19]$.

Although prior work has described the 'periventricular' and 'deep' spatial distributions of white matter lesions [20,21], no juxtacortical localization has been performed to date, and it is unknown whether such cortically proximal regional distributions differ in typical aging compared to AD. This information is critical in that it may provide new mechanisms for identifying individuals at risk for the development of $\mathrm{AD}$, as well as yield a clearer picture of the underlying pathologies of the disease, which is becoming increasingly recognized as a heterogeneous process with potential vascular contributions [22, 23]. In addition, associating WMSA with proximal cortex may be important for understanding the functional consequences of damages white matter as well as for designing and testing potential therapeutic interventions. The current understanding is that WMSA first appear in periventricular WM where tissue is most susceptible to hypoperfusion and likely progresses outwards toward the juxtacortical WM over time in 'normal' aging [9, 24], although regionally isolated WMSA exist as well. Several studies have demonstrated WMSA localized to parietal $[25,26]$ and periventricular regions $[18,27-30]$ as being indicators of $\mathrm{AD}$, yet other studies have found limited or no spatial differences between AD and controls in terms of distribution of WMSA [15, 31]. 
A potential reason for the discrepancies between studies may be the methodologies used to assess WMSA. In general, WMSA are rated using semi-quantitative measures such as the Fazekas scale [32] or they are automatically quantified with computational segmentation techniques. In either case, their spatial patterns are typically described as belonging to 'deep' versus 'periventricular' WM, sometimes further subdivided into the four main lobes of the brain [15, 33-35]. This has provided some information about the spatial distribution of tissue damage, yet the large volume of such descriptions likely results in regional heterogeneity within each lobar measurement, and additionally may miss critical areas in which WMSA may reflect specific focal functional consequence. In addition to the lack of understanding regarding the precise spatial accumulation of WMSA, it is unclear if certain regions accumulate more WMSA than other regions as the total amount of WMSA in the brain increases, and whether regional differences may differentiate cognitively healthy older adults form individuals with AD.

In the present study, we demonstrate a novel mapping procedure for the spatial assessment of WMSA based on proximity to 35 anatomically-based cortical regions [36, 37]. We use this technique to determine whether the global increase in WMSA reported for individuals with $\mathrm{AD}$ is due to preferential regional accumulation as well as whether spatial patterns in WMSA volume differ in AD compared to cognitively healthy older adults when accounting for global WMSA volume (i.e., does accumulation in certain regions scale disproportionately with global WMSA volume in AD compared to what is seen is typical aging). The overall findings in this study demonstrate spatial differences in WMSA accumulation in $\mathrm{AD}$ that are related to the conversion from mild cognitive impairment (MCI) to AD providing information linking specific regional accumulation of WMSA to the development of dementia.

\section{METHODS}

\section{Data}

Data used in the preparation of this article were obtained from the Alzheimer's Disease Neuroimaging Initiative (ADNI) database (http://adni.loni.usc.edu). The ADNI was launched in 2003 as a public-private partnership, led by Principal Investigator Michael W. Weiner, MD. The primary goal of ADNI has been to test whether serial MRI, positron emission tomography, other biological markers, and clinical and neuropsychological assessment can be combined to measure the progression of MCI and early AD. For up-todate information, see http://www.adni-info.org.

The present study used data from the ADNI-1 database that were processed locally at the time of analysis and were selected based on availability of T1-weighted, T2-weighted, and proton density (PD)-weighted images for WMSA processing. Three hundred and eight individuals were used in the following analyses; 127 of these had a diagnosis of AD, 107 were cognitively healthy age-matched older controls (OC), and 74 had a diagnosis of amnestic MCI (Table 1). ADNI-1 follows individuals every 6 months and so MCI individuals were stratified by ADNI- 1 into three groups based on their distance in time from AD conversion: those who were 6 months away from converting (MCI-6; $n=16$ ), those 12 months away from converting (MCI-12; $n=35$ ), and those 24 months away from converting 
(MCI-24; $n=23$ ). Data were selected based on the having sufficient quality to be processed by FreeSurfer and limited to datasets with available T1-weighted, T2-weighted, and proton density (PD)-weighted images for WMSA processing. Demographic data such as age, sex, years of education, history of hypertension, history of endocrine-metabolic disorder, and Mini-Mental State Examination (MMSE) scores were additionally acquired from the ADNI database (Table 1).

\section{MRI acquisition}

All data were acquired on a 1.5-T scanner at rigorously validated sites, which all followed a previously described standardized protocol [38]. The protocol included a high-resolution, T1-weighted sagittal volumetric magnetization prepared rapid gradient echo sequence and axial PD and/or T2-weighted fast spin echo sequence. The ADNI MRI core optimized the acquisition parameters of these sequences for each make and model of scanner included in the study. All scanner sites were required to pass a strict scanner validation test before being allowed to scan ADNI participants. Additionally, each scan of ADNI participants included a scan of the phantom, which was required to pass additional strict validation tests. For the purposes of this study, it is important to note that although T2/PD data were acquired at a lower resolution than the $\mathrm{T} 1$ data, the T2/PD sequences having a $5 \mathrm{~mm}$ slice thickness, identification of juxtacortical white matter was done solely with the $1 \mathrm{~mm}^{3} \mathrm{~T} 1$-weighted data through FreeSurfer's main recon-all processing stream. T2/PD data were then registered to the T1 data and partial volume correction was performed [39].

\section{MRI preprocessing}

Each individual's T1-weighted MRI was processed using FreeSurfer's main recon-all processing stream with a recently described extension for the segmentation of WMSAs [2]. Cortical reconstruction and volumetric segmentation was performed using FreeSurfer (http:// surfer.nmr.mgh.harvard.edu/, version 5.1). The technical details of these procedures are described in prior publications [36, 40-43]. Importantly, the FreeSurfer atlas used in the recon-all processing stream is based on manual labelings of 40 subjects distributed in age and pathology, with 10 each of young, middle-aged, and elderly subjects and an additional 10 with an in vivo diagnosis of probable AD. The explicit inclusion of pathological examples in the atlas makes it significantly more robust to neurodegeneration in morphometric studies. All processed data were manually inspected for accurate volumetric segmentation and quality of cortical boundary delineation.

\section{WMSA segmentation}

Images were processed with an automatic WMSA segmentation stream that has been previously described [2]. Briefly, this procedure registers T2-and PD-weighted images to an individual's T1-weighted image after it has been processed through FreeSurfer's recon-all stream. It then performs intensity normalization of all three modalities using a multimodal atlas and segments WMSA from normal-appearing white matter (NAWM) using a spatial array of multimodal Gaussian classifiers as well as individual-based heuristics. The principles implemented are similar to those used in the FreeSurfer automatic segmentation stream for normal brain structures, but it is important to note that this method is distinct from the 'WMHypointensity' segmentation method performed in the FreeSurfer recon-all 
processing stream which uses T1-weighted images alone. The use of three different imaging modalities with this newer method (more thoroughly described in Lindemer et al. [2]) provides more information on WM integrity and therefore allows for more accurate WMSA labeling. Given the use of multiple image types (T1/T2/PD), the signal may be 'hyper' or 'hypo' intense depending on the image modality, motivating our use of the term 'WMSA'.

Specifically, on T1-weighted images gray matter (GM) and cerebrospinal fluid (CSF) appear darker than WM, but WMSA appear darker than the surrounding NAWM. On T2-weighted imaging, CSF is the brightest followed by GM and then WM, but WMSA appear brighter than its surrounding NAWM. On PD-weighted imaging, GM is brighter than WM but WMSA appear brighter than surrounding NAWM.

The WM was divided into 70 regions of interest (ROIs) that encompassed the entire subcortical WM as well as the periventricular WM as described previously [44]. Briefly, the cortical parcellations defined by the FreeSurfer recon-all processing stream are used to subsequently assign a label to the underlying WM by the construction of a Voronoi diagram in the WM voxels based on distance to the nearest cortical parcellation label. Using a distance constraint of $5 \mathrm{~mm}$, each Voronoi polygon inherited the label of the cortical parcellation unit, yielding a complete labeling of the cerebral WM. As WMSA has been shown to be a generally symmetrical pathology [45] and we expected and then verified regional WMSA symmetry in this work, we therefore combined across hemispheres for a total of 35 final ROIs. Regional WMSA differences between diagnostic groups were first assessed using the raw WMSA volume as a metric. Due to the known decrease in WM volume with increasing age [44], the WMSA volume as a percent of the individual's nativespace ROI total WM was also calculated for each ROI. In this way, a clearer picture of 'functional' impairment may be disentangled, as it indicates how much healthy tissue remains in the region.

\section{Statistical analyses}

A general linear model (GLM) was implemented to test the difference in WMSA burden in each ROI between $\mathrm{AD}(n=127)$ and OC $(n=107)$ groups while controlling for age. This was performed using both raw WMSA volume and WMSA as a percent of each ROI. To insure that regional increases were not simply due to greater global WMSA volume, these GLMs were also conducted using regional WMSA burden normalized to the individual's total WMSA burden. In total, each ROI was tested for group differences four times using two different WMSA metrics (raw WMSA volume and WMSA\% of ROI) with and without normalization for total WMSA. Results are reported with and without correction for multiple comparisons using the false discovery rate (FDR) method [46], correcting for 140 tests. Since raw WMSA volume and WMSA as a percent of each ROI showed similar results when comparing AD to OC, the WMSA percent of each ROI was calculated for each MCI $(n=74)$ individual. A linear regression was performed on WMSA burdens in each ROI across the three MCI subgroups, and a linear hypothesis test was conducted to determine if there was a significant increase in WMSA burden in MCI individuals who were closer in time to $\mathrm{AD}$ conversion. 
Correction for global WMSA reduced the number of regions exhibiting group differences in WMSA volume between AD and OC. We therefore conducted secondary analyses to determine whether regional group by global WMSA burden interactions could be detected which would indicate differential scaling of regional WMSA with global WMSA between the groups suggesting differential regional damage propensity.

\section{RESULTS}

\section{Demographics}

Demographic information for the individuals in each of the diagnostic groups is listed in Table 1. As determined by a one-way ANOVA with post-hoc pairwise comparisons, groups did not differ significantly in age or years of education, but the AD group had significantly higher total WMSA $(p<0.001)$. As expected due to the use of MMSE score in diagnoses, $\mathrm{AD}$ individuals had lower scores over $\mathrm{OC}$ and all $\mathrm{MCI}$ subgroups, and $\mathrm{OC}$ were significantly higher than all MCI subgroups. Chi-squared goodness-of-fit tests demonstrated that there was no significant difference in group distributions of sex, history of hypertension, hypercholesteremia, or endocrine-metabolic disorders. In the OC group, age was significantly correlated with total WMSA burden $(\mathrm{R}=0.3299, p<0.001)$ but not in $\mathrm{AD}(\mathrm{R}=$ $0.1369, p=0.1248)$, nor in the combined group of MCI individuals $(\mathrm{R}=0.1512, p=$ $0.1985)$. There was no significant group by global WMSA interaction with age. Due to these findings, all further group comparisons with WMSA were controlled for age.

\section{Spatial differences in raw WMSA volume}

Out of the 35 ROIs, 26 demonstrated significantly greater raw WMSA volume in AD compared to OC ( $p<0.05$ uncorrected; Fig. 1, Top Left). All of these survived FDR correction except for the banks of the superior temporal sulcus, lateral orbitofrontal, precuneus, and superior temporal WM $(p=0.02)$. After normalizing the WMSA volume in each ROI by each individual's total WMSA burden, nine regions remained significant at $p<$ 0.05 (Fig. 1, bottom left). Of these, fusiform, inferior parietal, inferior temporal, pars orbitalis, rostral middle frontal, and supramarginal WM survived FDR correction. Insular and periventricular WMSA showed greater normalized WMSA volumes in OC compared to $\mathrm{AD}$, but only insular WM survived FDR correction.

There was a significant group by global WMSA interaction for WMSA within several regions including cuneus, fusiform, inferior parietal, inferior temporal, lateral occipital, middle temporal, periventricular and frontal pole WM $(p<0.05)$. This indicates that increasing global WMSA is linked to a greater accumulation of local WMSA in these regions compared to the accumulation in $\mathrm{OC}$ demonstrating the differential lesion properties in these groups.

Follow-up analyses assessed whether spatial differences were seen when examining only individuals with hypertension as well as only individuals without hypertension in each diagnostic group. In AD individuals with hypertension, there was a significantly higher WMSA burden in caudal anterior cingulate WM than in those without hypertension. In OC individuals with hypertension, there was a significantly higher WMSA burden in the banks 
of the superior temporal sulcus, lateral orbitofrontal, middle temporal, precuneus, superior temporal, supramarginal, and periventricular WM over those without hypertension.

\section{Spatial Differences in WMSA as a percent of total ROI WM}

Measures used in the prior results were based on raw WMSA within a region. However, WMSA volumes corrected for regional total tissue volume is likely a more relevant marker of the degree of regional damage and compromise. We therefore repeated the ROI comparisons using a corrected measure of regional WMSA as a \% of total WM volume in the region. Results remained essentially unchanged except in precuneus and superior temporal regions, which no longer reached significance (Fig. 1, top right). Of the 24 significant ROIs, only lateral orbitofrontal, posterior cingulate, and superior parietal did not survive FDR correction. We next performed a secondary normalization of the WMSA percent measure to each individual's total WMSA (normalization for global WMSA) and found 8 ROIs to have significantly higher WMSA in AD, and insular WM to have higher WMSA in OC (Fig. 1, bottom right). Of these, only inferior parietal and frontal pole regions did not survive FDR correction.

When comparing individuals with and without hypertension in each diagnostic group, only precuneus WM showed a greater WMSA/total WMSA ratio in the OC group with hypertension over the OC group without hypertension.

\section{Spatial WMSA and MCl time to conversion}

Of the 35 ROIs tested, all but four exhibited the highest mean WMSA in the MCI-6 group (the MCI closest to conversion) relative to the other groups. A linear regression demonstrated that there was a significant relationship between regional WMSA burden and time-to-AD-conversion in fusiform, inferior temporal, pericalcarine, rostral middle frontal, and superior frontal WM ( $p<0.05$ uncorrected) (Fig. 2). After normalizing for total WMSA, only inferior temporal and rostral middle frontal regions exhibited a significant relationship with time-to-AD-conversion.

\section{DISCUSSION}

We demonstrate here for the first time a set of juxtacortical WM regions where a greater WMSA volume is found in individuals with AD, where the scaling of WMSA with global WMSA is different in patients with AD compared to OC, and where larger volumes of WMSA are indicative of individuals with MCI who are temporally close to conversion. These results provide unique insight into the pathological basis of AD dementia and demonstrate the utility of the regional WMSA mapping procedure based on proximity to specific cortical regions. Future work will investigate if this regionally-specific WMSA manifestation is linked to distinct functional and clinical consequences. In addition to the regional analyses, we explored the relationship between regional and global WMSA volume and also measure WMSA as a percent of the ROI's total WM to account for WM tissue atrophy that is known to occur in aging and dementia [44]. While prior studies have demonstrated regional differences in periventricular $[27,28]$ and parietal $[25,26]$ WMSA between the two diagnostic groups, we provide evidence for more widespread increases in 
WMSA in AD that include temporal and frontal WM, and that some of these regions also show increases in WMSA as individuals with MCI are closer to AD conversion. It has been previously established that individuals with AD have greater global WMSA burdens [2, 27, $28,47,48]$, and to ensure that this factor is not solely responsible for any regional increases, our technique in which we normalize for global WMSA describes what we believe to be true regional increases in cuneus, inferior temporal, middle frontal, and inferior parietal WM. Based on our findings, we hypothesize that the spatial distribution of WMSA may be important to the understanding of the pathological processes that occur separately from normal aging during the development of AD. This is supported by the relationship between increasing regional WMSA burden and decreasing time-to-AD-conversion in MCI individuals. While the histopathological profile of WMSA presents as complex and heterogeneous and includes gliosis and demyelination, WMSA are generally regarded as a vascular pathology [5, 6]. Furthermore, studies that compare the histopathological profiles of WMSA between $\mathrm{AD}$ and $\mathrm{OC}$ individuals have demonstrated that they are similarly comprised of lacunes, arteriosclerosis, cerebral amyloid angiopathy, and microinfarcts [49]. Individuals with $\mathrm{AD}$, however, demonstrate more marked deep WM demyelination as well as nearly doubled thickness of the adventitia of deep WM arteries over OC individuals [4] and WM atherosclerosis as well as WMSA burden are associated with higher Braak [50] scores in aging populations [8]. Thus, our findings along with previous findings provide evidence that vascular pathologies may be contributing factors to AD development, acting either independently from more classically known $\mathrm{AD}$ pathologies or due to a common upstream process. While some studies have found WMSA to be associated with classic AD pathologies such as cerebral amyloid [51], others have found WMSA and cerebral amyloid to be independent predictors of $\mathrm{AD} \mathrm{[14]} \mathrm{and} \mathrm{that} \mathrm{vascular} \mathrm{risk} \mathrm{factors} \mathrm{are} \mathrm{associated} \mathrm{with}$ WMSA independent of CSF amyloid levels [52]. The findings of increased regional WMSA volume independent of global volume suggests that there is something unique about $\mathrm{AD}$ that results in this regional WMSA accumulation, and that WMSA do not simply represent a comorbid vascular condition that is found to be heightened in individuals with AD.

As we demonstrate in our analyses in which we normalize for total WMSA, regional differences between groups are strongly influenced by total WMSA burden. Prior to normalization for global WMSA burden, there were widespread regions where AD had greater WMSA volume compared to OC including medial frontal, postcentral, and precentral WM. This finding perhaps demonstrates a vascular comorbidity of AD that is expressed over cognitively healthy older controls but which is not a unique component of the AD disease process itself. After normalization for global WMSA however, 9 out of 35 ROIs remain significantly greater in WMSA volume in AD including inferior temporal, middle frontal, inferior parietal, and cuneus WM. We believe that this is a critical concept in understanding the contribution of WMSA to AD in that it isolates regional differences independent of global WMSA burden. To our knowledge, with the exception of parietal WMSA [25, 26], these regions have not been reported to demonstrate higher WMSA in AD and we attribute these findings to our methodology which normalizes for global WMSA burden. WMSA in temporal WM fit into the classic picture of AD that demonstrates marked cortical changes in relation to memory loss symptoms, but whether these are two separate processes or part of the same pathological pathway is currently unknown. The increase in 
frontal WMSA in AD may explain some of the executive dysfunction seen in AD [53,54] which is highly dependent on frontal lobe function. Furthermore, others have shown that increased WMSA anywhere in the brain is associated with greater executive dysfunction [55]. Interestingly, we find that normalizing for total WMSA brings out a significant increase in periventricular and insular WMSA in cognitively healthy older controls over individuals with $\mathrm{AD}$, which to our knowledge has not been previously demonstrated. In fact, several studies have reported greater WMSA in periventricular regions in individuals with $\mathrm{AD}[18,27,28]$, but these studies did not control for global WMSA and therefore may have been detecting global WMSA increases that were most obvious in periventricular WM as this is where the majority of WMSA occur in the course of aging. We interpret this finding to mean that WMSA preferentially accumulate in deep regions where perfusion is low during the course of normal aging such as periventricular and insular WM, but that the WMSA that accumulate elsewhere in AD may be due to a selective type of vascular dysfunction that is unique to $\mathrm{AD}$ and not found in general vascular conditions.

Our results indicate that as $\mathrm{MCI}$ individuals are closer in time to AD conversion, WMSA burden increases in regions that demonstrate higher WMSA in AD over OC. In our linear regression analyses, we demonstrate that the regions showing a statistically significant relationship between WMSA and time-to-AD-conversion are limited to the inferior temporal and the frontal WM. We note that this does not include parietal WM, which did show significantly increased WMSA in AD individuals over the OC group, and has also been shown by other groups to be predictive of AD conversion [26]. We interpret this finding to suggest that perhaps parietal WMSA follow a different time course than temporal and frontal WMSA, as we see a significant relationship in these regions within the two years prior to AD conversion, but the duration of MCI can be much longer depending on the individual. Additionally, with greater sample sizes within each of the MCI subgroups, significant relationships may be revealed as trend-level relationships are seen in parietal WMSA but were not statistically significant, and we acknowledge the small MCI sample sizes as a limitation of the present study which likely contributes to the high variance in MCI subgroups. Taken together, however, these findings indicate that regionally-specific WMSA may in fact be a critical component of $\mathrm{AD}$ development as their progression can be viewed prior to $\mathrm{AD}$ onset and is directly related to time-to-conversion.

Interestingly, the regions demonstrating higher WMSA in AD as well as a relationship in the MCI individuals regardless of the WMSA metric used and after normalizing for total WMSA align to known cerebrovascular boundary zones (also known as watershed regions; Supplementary Figure 1), which are areas that are at the border of blood supply zones from two different major cerebral arteries and are thus most susceptible to hypoperfusion [56]. Specifically, these include superior frontal, supramarginal, inferior temporal, and cuneus regions. Furthermore, these regions are in close proximity to cortical regions in which cerebral blood flow (CBF) is known to decrease with advancing age [57]. The relationship between reductions in WM integrity and reductions in cortical CBF has also been previously demonstrated $[58,59]$, supporting the idea that the effects seen in these regions may be linked to neighboring cortical perfusion changes. We therefore interpret our findings to suggest that there may be vascular changes in $\mathrm{AD}$ that are at least regionally accelerated relative to global changes in comparison to those seen in normal aging. Our initial findings 
with WMSA, hypertension, and AD diagnosis suggest that this relationship is complex and future work will examine whether or not there are interactive effects between vascular risk factors and a clinical diagnosis of AD on the manifestation of WMSA, and how these effects are spatially represented.

Overall, the current data demonstrate a regionally stereotyped increase in WMSA burden with increasing overall burden in cognitively healthy older adults as well as adults with AD. There is a widespread increase in WMSA in individuals with AD, although the spatial extent of these increases is reduced when regional WMSA is normalized for total WMSA burden and are limited to inferior temporal, inferior parietal, cuneus, and middle frontal WM. Our results indicate that regionally-specific WMSA may be an important pathological component of AD development and we hypothesize that these WMSA may be linked to a vascular component of disease progression.

\section{Supplementary Material}

Refer to Web version on PubMed Central for supplementary material.

\section{Acknowledgments}

Support for this work was provided by the NIH Neuroimaging Training Program Grant \#T32 EB001680 from the National Institute of Biomedical Imaging and Bioengineering (NIBIB), a training grant from the NIH Blueprint for Neuro-science Research (T90DA022759/R90DA023427), and National Institute for Nursing Research R01NR010827. Additional support was provided in part by the National Institute for Biomedical Imaging and Bioengineering (P41EB015896, R01EB006758, R21EB018907, R01EB019956), the National Institute on Aging (5R01AG008122, R01AG016495), the National Institute of Diabetes and Digestive and Kidney Diseases (1-R21DK-108277-01), the National Institute for Neurological Disorders and Stroke (R01NS0525851, R21NS072652, R01NS070963, R01NS083534, 5U01NS086625), and was made possible by the resources provided by Shared Instrumentation Grants 1S10RR023401, 1S10RR019307, and 1S10RR023043. Additional support was provided by the NIH Blueprint for Neuroscience Research (5U01-MH093765), part of the multi-institutional Human Connectome Project.

Data collection and sharing for this project was funded by the Alzheimer's Disease Neuroimaging Initiative (ADNI) (National Institutes of Health Grant U01 AG024904) and DOD ADNI (Department of Defense award number W81XWH-12-2-0012). ADNI is funded by the National Institute on Aging, the National Institute of Biomedical Imaging and Bioengineering, and through generous contributions from the following: AbbVie, Alzheimer's Association; Alzheimer's Drug Discovery Foundation; Araclon Biotech; BioClinica, Inc.; Biogen; Bristol-Myers Squibb Company; CereSpir, Inc.; Cogstate; Eisai Inc.; Elan Pharmaceuticals, Inc.; Eli Lilly and Company; EuroImmun; F. Hoffmann-La Roche Ltd and its affiliated company Genentech, Inc.; Fujirebio; GE Healthcare; IXICOLtd.; Janssen Alzheimer Immunotherapy Research \& Development, LLC.; Johnson \& Johnson Pharmaceutical Research \& Development LLC.; Lumosity; Lundbeck; Merck \& Co., Inc.; Meso Scale Diagnostics, LLC.; NeuroRx Research; Neurotrack Technologies; Novartis Pharmaceuticals Corporation; Pfizer Inc.; Piramal Imaging; Servier; Takeda Pharmaceutical Company; and Transition Therapeutics. The Canadian Institutes of Health Research is providing funds to support ADNI clinical sites in Canada. Private sector contributions are facilitated by the Foundation for the National Institutes of Health (http://www.fnih.org). The grantee organization is the Northern California Institute for Research and Education, and the study is coordinated by the Alzheimer's Therapeutic Research Institute at the University of Southern California. ADNI data are disseminated by the Laboratory for Neuro Imaging at the University of Southern California.

Authors' disclosures available online (http://j-alz.com/manuscript-disclosures/16-1057r2).

\section{References}

1. Fazekas F, Niederkron K, Schmidt R, Offenbacher H, Horner S, Bertha G, Lechner H. White matter signal abnormalities in normal individuals: Correlation with carotid ultrasonography, cerebral blood flow measurements, and cerebrovascular risk factors. Stroke. 1988; 19:1285-1288. [PubMed: 3051534] 
2. Lindemer ER, Salat DH, Smith EE, Nguyen K, Fischl B, Greve DN. White matter signal abnormality quality differentiates mild cognitive impairment that converts to Alzheimer's disease from nonconverters. Neurobiol Aging. 2015; 36:2447-2457. [PubMed: 26095760]

3. Gouw A, Seewann A, van der Flier WM, Barkhof F, Rozemuller AM, Scheltens P, Geurts JJG. Heterogeneity of small vessel disease: A systematic review of MRI and histopathology correlations. J Neurol Neurosurg Psychiatry. 2011; 82:126-135. [PubMed: 20935330]

4. Scheltens P, Barkhof F, Leys D, Wolters EC, Ravid R, Kamphorst W. Histopathologic correlates of white matter changes on MRI in Alzheimer's disease and normal aging. Neurology. 1995; 45:883888. [PubMed: 7746401]

5. Benedictus MR, Binnewijzend MAA, Kuijer JPA, Steenwijk MD, Versteeg A, Vrenken H, Scheltens P, Barkhof F, van der Flier WM, Prins ND. Brain volume and white matter hyperintensities as determinants of cerebral blood flow in Alzheimer's disease. Neurobiol Aging. 2014; 35:2665-2670. [PubMed: 25018106]

6. Wardlaw JM, Smith C, Dichgans M. Mechanisms of sporadic cerebral small vessel disease: Insights from neuroimaging. Lancet Neurol. 2013; 12:483-497. [PubMed: 23602162]

7. Iadecola C. The pathobiology of vascular dementia. Neuron. 2013; 80:844-866. [PubMed: 24267647]

8. Erten-Lyons D, Woltjer R, Kaye J, Mattek N, Dodge HH, Green S, Tran H, Howieson DB, Wild K, Silbert LC. Neuropathologic basis of white matter hyperintensity accumulation with advanced age. Neurology. 2013; 81:977-983. [PubMed: 23935177]

9. de Leeuw FE, de Groot JC, Achten E, Oudkerk M, Ramos LM, Heijboer R, Hofman A, Jolles J, van Gijn J, Breteler MM. Prevalence of cerebral white matter lesions in elderly people: A population based magnetic resonance imaging study. The Rotterdam Scan Study. J Neurol Neurosurg Psychiatry. 2001; 70:9-14. [PubMed: 11118240]

10. Longstreth WT Jr. Brain abnormalities in the elderly: Frequency and predictors in the United States (the Cardiovascular Health Study) Cardiovascular Health Study Collaborative Research Group. J Neural Transm Suppl. 1998; 53:9-16. [PubMed: 9700642]

11. Debette S, Markus HS. The clinical importance of white matter hyperintensities on brain magnetic resonance imaging: Systematic review and meta-analysis. BMJ. 2010; 341:c3666. [PubMed: 20660506]

12. Carmichael O, Schwarz C, Drucker D, Fletcher E, Harvey D, Beckett L, Jack CR, Weiner M, DeCarli C. Longitudinal changes in white matter disease and cognition in the first year of the Alzheimer disease neuroimaging initiative. Arch Neurol. 2010; 67:1370-1378. [PubMed: 21060014]

13. Maillard P, Carmichael O, Fletcher E, Reed B, Mungas D, DeCarli C. Coevolution of white matter hyperintensities and cognition in the elderly. Neurology. 2012; 79:442-448. [PubMed: 22815562]

14. Provenzano F, Muraskin J, Tosto G, Narkhede A, Wasserman BT, Griffith EY, Guzman V, Meier IB, Zimmerman ME, Brickman AM. White matter hyperintensities and cerebral amyloidosis: Necessary and sufficient for clinical expression of Alzheimer disease? JAMA Neurol. 2013; 70:455-461. [PubMed: 23420027]

15. Chen Y-F, Wang H, Chu Y, Huang Y-C, Su M-Y. Regional quantification of white matter hyperintensity in normal aging, mild cognitive impairment, and Alzheimer's disease. Dement Geriatr Cogn Disord. 2006; 22:177-184. [PubMed: 16912479]

16. Tosto G, Zimmerman ME, Carmichael OT, Brickman AM. Predicting aggressive decline in mild cognitive impairment: The importance of white matter hyperintensities. JAMA Neurol. 2014; 71:872-877. [PubMed: 24821476]

17. Brickman AM, Muraskin J, Zimmerman ME. Structural neuroimaging in Alzheimer's disease: Do white matter hyperintensities matter? Dialogues Clin Neurosci. 2009; 11:181-190. [PubMed: 19585953]

18. de Groot JC, de Leeuw FE, Oudkerk M, Van Gijn J, Hofman A, Jolles J, Breteler MMB. Periventricular cerebral white matter lesions predict rate of cognitive decline. Ann Neurol. 2002; 52:335-341. [PubMed: 12205646]

19. Frisoni GB, Galluzzi S, Pantoni L, Filippi M. The effect of white matter lesions on cognition in the elderly - small but detectable. Nat Clin Pr Neurol. 2007; 3:620-627. 
20. DeCarli C, Fletcher E, Ramey V, Harvey D, Jagust WJ. Anatomical mapping of white matter hyper-intensities (WMH): Exploring the relationships between periventricular WMH, deep WMH, and total WMH burden. Stroke. 2005; 36:50-55. [PubMed: 15576652]

21. Wen W, Sachdev P. The topography of white matter hyperintensities on brain MRI in healthy 60 - to 64-year-old individuals. Neuroimage. 2004; 22:144-154. [PubMed: 15110004]

22. Kawas CH, Kim RC, Sonnen JA, Bullain SS, Trieu T. Multiple pathologies are common and related to dementia in the oldest-old. Neurology. 2015; 85:535-542. [PubMed: 26180144]

23. Toledo JB, Arnold SE, Raible K, Brettschneider J, Xie SX, Grossman M, Monsell SE, Kukull WA, Trojanowski JQ. Contribution of cerebrovascular disease in autopsy confirmed neurodegenerative disease cases in the National Alzheimer's Coordinating Centre. Brain. 2013; 136:2697-2706. [PubMed: 23842566]

24. Wardlaw JM, Valdés Hernández MC, Muñoz-Maniega S. What are white matter hyperintensities made of? Relevance to vascular cognitive impairment. J Am Heart Assoc. 2015; 4:1140.

25. Brickman AM, Provenzano FA, Muraskin J, Manly JJ, Blum S, Apa Z, Stern Y, Brown TR, Luchsinger JA, Mayeux R. Regional white matter hyperintensity volume, not hippocampal atrophy, predicts incident Alzheimer disease in the community. Arch Neurol. 2012; 69:1621-1627. [PubMed: 22945686]

26. Brickman AM, Zahodne LB, Guzman VA, Narkhede A, Meier IB, Griffith EY, Provenzano FA, Schupf N, Manly JJ, Stern Y, Luchsinger JA, Mayeux R. Reconsidering harbingers of dementia: Progression of parietal lobe white matter hyperintensities predicts Alzheimer's disease incidence. Neurobiol Aging. 2015; 36:27-32. [PubMed: 25155654]

27. Yoshita M, Fletcher E, Harvey D, Ortega M, Martinez O, Mungas DM, Reed BR, DeCarli CS. Extent and distribution of white matter hyperintensities in normal aging, MCI, and AD. Neurology. 2006; 67:2192-2198. [PubMed: 17190943]

28. Barber R, Scheltens P, Gholkar A, Ballard C, McKeith I, Ince P, Perry R, O’Brien J. White matter lesions on magnetic resonance imaging in dementia with Lewy bodies, Alzheimer's disease, vascular dementia, and normal aging. J Neurol Neurosurg Psychiatry. 1999; 67:66-72. [PubMed: 10369824]

29. Prins ND, van Dijk EJ, den Heijer T, Vermeer SE, Koudstaal PJ, Oudkerk M, Hofman A, Breteler MMB. Cerebral white matter lesions and the risk of dementia. Arch Neurol. 2004; 61:1531-1534. [PubMed: 15477506]

30. Matsubayashi K, Shimada K, Kawamoto A, Ozawa T. Incidental brain lesions on magnetic resonance imaging and neurobehavioral functions in the apparently healthy elderly. Stroke. 1992; 23:175-180. [PubMed: 1561644]

31. Coutu J-P, Goldblatt A, Rosas HD, Salat DH. White matter changes are associated with ventricular expansion in aging, mild cognitive impairment, and Alzheimer's disease. J Alzheimers Dis. 2015; 49:329-342.

32. Fazekas F, Chawluk JB, Alavi A. MR signal abnormalities at $1.5 \mathrm{~T}$ in Alzheimer's dementia and normal aging. Am J Neuroradiol. 1987; 8:421-426.

33. Prasad K, Wiryasaputra L, Ng A, Kandiah N. White matter disease independently predicts progression from mild cognitive impairment to alzheimer's disease in a clinic cohort. Dement Geriatr Cogn Disord. 2011; 31:431-434. [PubMed: 21757908]

34. Kozachuk WE, DeCarli C, Schapiro MB, Wagner EE, Rapoport SI, Horwitz B. White matter hyperintensities in dementia of Alzheimer's type and in healthy subjects without cerebrovascular risk factors. A magnetic resonance imaging study. Arch Neurol. 1990; 47:1306-1310. [PubMed: 2252447]

35. Gootjes L, Teipel SJ, Zebuhr Y, Schwarz R, Leinsinger G, Scheltens P, Möller H-J, Hampel H. Regional distribution of white matter hyperintensities in vascular dementia, Alzheimer's disease and healthy aging. Dement Geriatr Cogn Disord. 2004; 18:180-188. [PubMed: 15211074]

36. Fischl B, Van Der Kouwe A, Destrieux C, Halgren E, Segonne F, Salat DH, Busa E, Seidman LJ, Goldstein J, Kennedy D, Caviness V, Makris N, Rosen B, Dale AM. Automatically parcellating the human cerebral cortex. Cereb Cortex. 2004; 14:11-22. [PubMed: 14654453]

37. Destrieux C, Fischl B, Dale A, Halgren E. Automatic parcellation of human cortical gyri and sulci using standard anatomical nomenclature. Neuroimage. 2010; 53:1-15. [PubMed: 20547229] 
38. Jack CR, Bernstein MA, Fox NC, Thompson P, Alexander G, Harvey D, Borowski B, Britson PJ, Whitwell JL, Ward C, Dale AM, Felmlee JP, Gunter JL, Hill DLG, Killiany R, Schuff N, FoxBosetti S, Lin C, Studholme C, DeCarli CS, Krueger G, Ward HA, Metzger GJ, Scott KT, Mallozzi R, Blezek D, Levy J, Debbins JP, Fleisher AS, Albert M, Green R, Bartzokis G, Glover G, Mugler J, Weiner MW. The Alzheimer's Disease Neuroimaging Initiative (ADNI): MRI methods. J Magn Reson Imaging. 2008; 27:685-691. [PubMed: 18302232]

39. Greve DN, Fischl B. Accurate and robust brain image alignment using boundary-based registration. Neuroimage. 2009; 48:63-72. [PubMed: 19573611]

40. Dale AM, Fischl B, Sereno MI. Cortical surface-based analysis. I. Segmentation and surface reconstruction. Neuroimage. 1999; 9:179-194. [PubMed: 9931268]

41. Fischl B, Liu A, Dale AM. Automated manifold surgery: Constructing geometrically accurate and topologically correct models of the human cerebral cortex. IEEE Trans Med Imaging. 2001; 20:70-80. [PubMed: 11293693]

42. Fischl B, Salat DH, Busa E, Albert M, Dieterich M, Haselgrove C, van der Kouwe A, Killiany R, Kennedy D, Klaveness S, Montillo A, Makris N, Rosen B, Dale AM. Whole brain segmentation: Automated labeling of neuroanatomical structures in the human brain. Neuron. 2002; 33:341-355. [PubMed: 11832223]

43. Fischl B, Salat DH, Van Der Kouwe A, Makris N, Segonne F, Quinn BT, Dale AM. Sequenceindependent segmentation of magnetic resonance images. Neuroimage. 2004; 23:S69-S84. [PubMed: 15501102]

44. Salat DH, Greve DN, Pacheco JL, Quinn BT, Helmer KG, Buckner RL, Fischl B. Regional white matter volume differences in nondemented aging and Alzheimer's disease. Neuroimage. 2009; 44:1247-1258. [PubMed: 19027860]

45. Wardlaw JM, Smith EE, Biessels GJ, Cordonnier C, Fazekas F, Frayne R, Lindley RI, O’Brien JT, Barkhof F, Benavente OR, Black SE, Brayne C, Breteler M, Chabriat H, Decarli C, de Leeuw F-E, Doubal F, Duering M, Fox NC, Greenberg S, Hachinski V, Kilimann I, Mok V, Oostenbrugge R, Van Pantoni L, Speck O, Stephan BCM, Teipel S, Viswanathan A, Werring D, Chen C, Smith C, van Buchem M, Norrving B, Gorelick PB, Dichgans M. Neuroimaging standards for research into small vessel disease and its contribution to ageing and neurodegeneration. Lancet Neurol. 2013; 12:822-838. [PubMed: 23867200]

46. Genovese C, Wasserman L. Operating characteristics and extensions of the false discovery rate procedure. J R Stat Soc Ser B Stat Methodol. 2002; 64:499-517.

47. Bowen BC, Barker WW, Loewenstein DA, Sheldon J, Duara R. MR signal abnormalities in memory disorder and dementia. AJR Am J Roentgenol. 1990; 11:283-290.

48. Tanabe JL, Ezekiel F, Jagust WJ, Reed BR, Norman D, Schuff N, Weiner MW, Chui H, Fein G. Magnetization transfer ratio of white matter hyperintensities in subcortical ischemic vascular dementia. AJNR Am J Neuroradiol. 1999; 20:839-844. [PubMed: 10369354]

49. Mcaleese KE, Firbank M, Hunter D, Sun L, Hall R, Neal JW, Mann DMA, Esiri M, Jellinger KA, O'Brien JT, Attems J. Magnetic resonance imaging of fixed post mortem brains reliably reflects subcortical vascular pathology of frontal, parietal and occipital white matter. Neuropathol Appl Neurobiol. 2013; 39:485-497. [PubMed: 23075072]

50. Braak H, Braak E. Neuropathological stageing of Alzheimer-related changes. Acta Neuropathol. 1991; 82:239-259. [PubMed: 1759558]

51. Marnane M, Al-Jawadi OO, Mortazavi S, Pogorzelec KJ, Wang BW, Feldman HH, Hsiung GYR. Periventricular hyperintensities are associated with elevated cerebral amyloid. Neurology. 2016; 86:535-543. [PubMed: 26747881]

52. Scott JA, Braskie MN, Tosun D, Thompson PM, Weiner M, DeCarli C, Carmichael OT. Cerebral amyloid and hypertension are independently associated with white matter lesions in elderly. Front Aging Neurosci. 2015; 7:1-9. [PubMed: 25653617]

53. Swanberg MM, Tractenberg RE, Mohs R, Thal LJ, Cummings JL. Executive dysfunction in Alzheimer disease. Arch Neurol. 2004; 61:556-560. [PubMed: 15096405]

54. Peavy GM, Salmon DP, Edland SD, Tam S, Hansen LA, Masliah E, Galasko D, Hamilton JM. Neuropsychiatric features of frontal lobe dysfunction in autopsy-confirmed patients with lewy 
bodies and "pure" alzheimer disease. Am J Geriatr Psychiatry. 2013; 21:509-519. [PubMed: 23567425]

55. Tullberg M, Fletcher E, DeCarli C, Mungas D, Reed BR, Harvey DJ, Weiner MW, Chui HC, Jagust WJ. White matter lesions impair frontal lobe function regardless of their location. Neurology. 2004; 63:246-253. [PubMed: 15277616]

56. Moody DM, Bell MA, Challa VR. Features of the cerebral vascular pattern that predict vulnerability to perfusion or oxygenation deficiency: An anatomic study. Am J Neuroradiol. 1990; 11:431-439. [PubMed: 2112304]

57. Chen JJ, Rosas HD, Salat DH. Age-associated reductions in cerebral blood flow are independent from regional atrophy. Neuroimage. 2011; 55:468-478. [PubMed: 21167947]

58. Chen JJ, Rosas HD, Salat DH. The relationship between cortical blood flow and sub-cortical whitematter health across the adult age span. PLoS One. 2013; 8:e56733. [PubMed: 23437228]

59. Brickman AM, Zahra A, Muraskin J, Steffener J, Holland CM, Habeck C, Borogovac A, Ramos MA, Brown TR, Asllani I, Stern Y. Reduction in cerebral blood flow in areas appearing as white matter hyperintensities on magnetic resonance imaging. Psychiatry Res. 2009; 172:117-120. [PubMed: 19324534] 


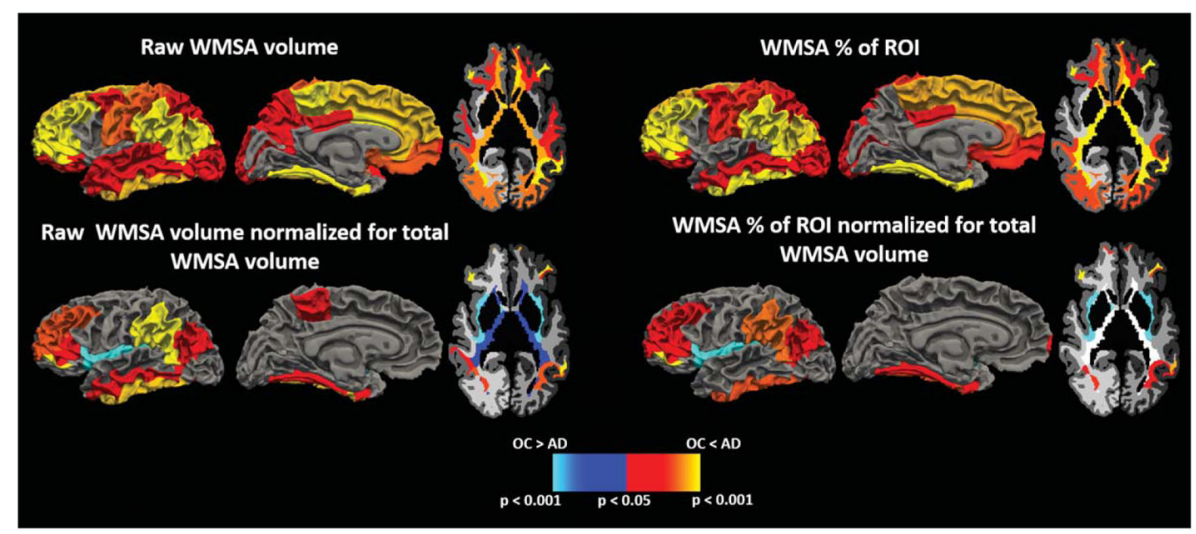

Fig. 1.

Regions demonstrating group differences in WMSA between AD and OC individuals when using raw WMSA volume as a metric (top left), raw WMSA volume normalized to total WMSA volume (bottom left), WMSA percent of ROI WM (top right), and WMSA percent of ROI WM normalized for total WMSA volume (bottom right). Warm colors indicate where AD have higher WMSA burdens than OC, and cool colors represent where OC have higher WMSA burdens than AD. 


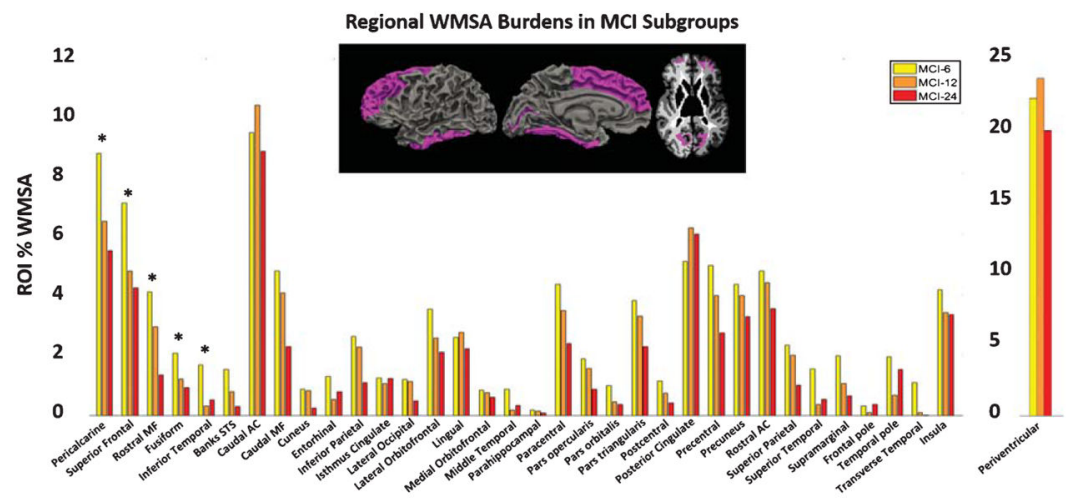

Fig. 2.

Mean WMSA burdens as a percent of total ROI WM volume in all 35 ROIs in the three MCI subgroups. Inset surface and volume images represent regions showing a significant relationship between WMSA and time-to-AD-conversion which are also starred on the graph $(* p<0.05)$. 


\section{를 \\ 골}

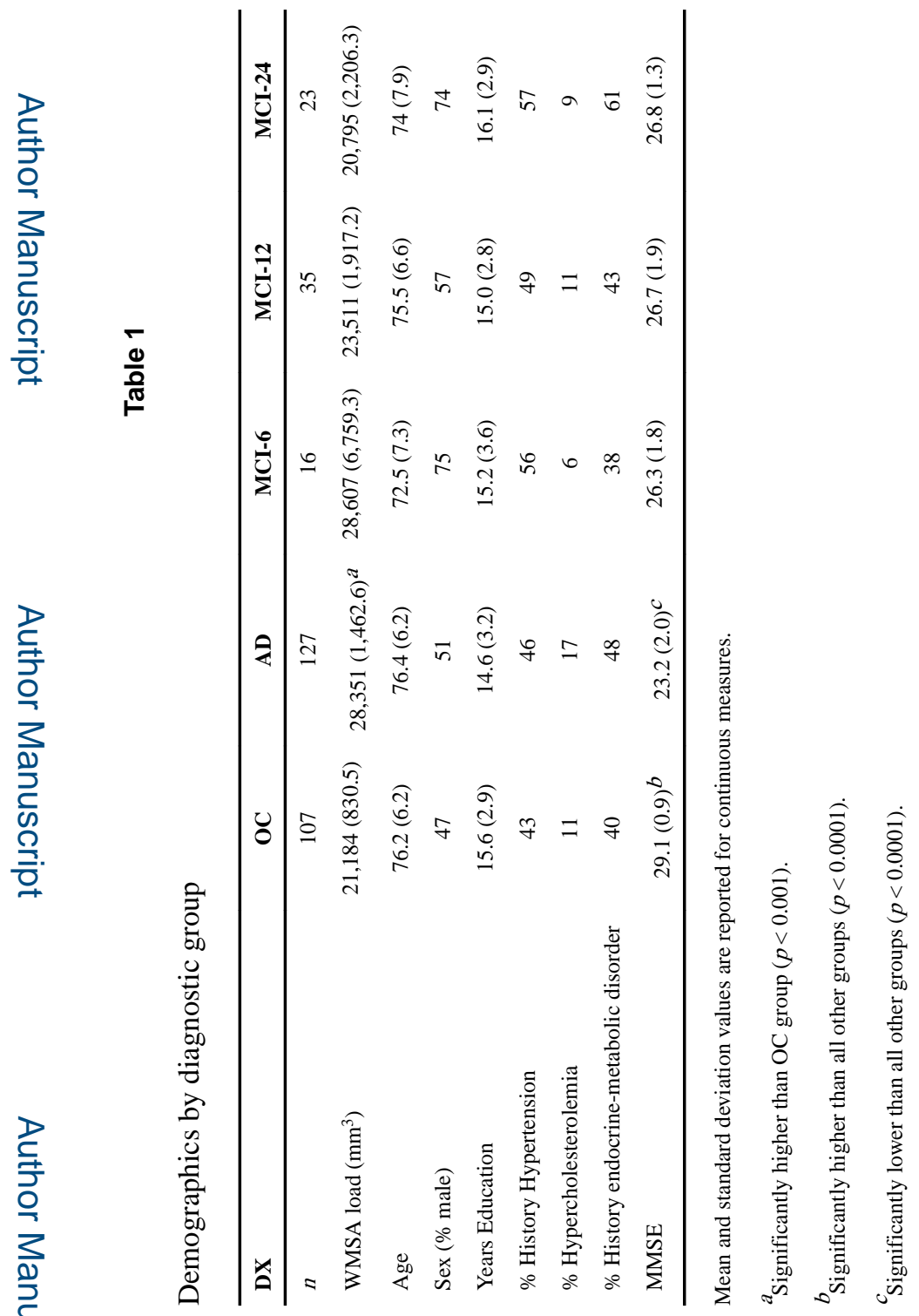

